\title{
A Novel Approach to Fault Diagnosis in Multicircuit Transmission Lines Using Fuzzy ARTmap Neural Networks
}

\author{
Raj K. Aggarwal, Senior Member, IEEE, Q. Y. Xuan, Allan T. Johns, Senior Member, IEEE,
} Furong $\mathrm{Li}$, and Allen Bennett

\begin{abstract}
The work described in this paper addresses the problems of fault diagnosis in complex multicircuit transmission systems, in particular those arising due to mutual coupling between the two parallel circuits under different fault conditions; the problems are compounded by the fact that this mutual coupling is highly variable in nature. In this respect, artificial intelligence (AI) technique provides the ability to classify the faulted phase/phases by identifying different patterns of the associated voltages and currents. In this paper, a Fuzzy ARTmap (Adaptive Resonance Theory) neural network is employed and is found to be well-suited for solving the complex fault classification problem under various system and fault conditions. Emphasis is placed on introducing the background of AI techniques as applied to the specific problem, followed by a description of the methodology adopted for training the Fuzzy ARTmap neural network, which is proving to be a very useful and powerful tool for power system engineers. Furthermore, this classification technique is compared with a Neural Network (NN) technique based on the error backpropagation (EBP) training algorithm, and it is shown that the former technique is better suited for solving the fault diagnosis problem in complex multicircuit transmission systems.
\end{abstract}

Index Terms-Artificial intelligence, fault classifier, fault diagnosis, fuzzy ARTmap, neural networks.

\section{INTRODUCTION AND BACKGROUND}

B ECAUSE of environmental and regulatory concerns, the growth of electric power transmission facilities is restricted; as a consequence, transmission bottlenecks, underutilization, and uncontrollable usage of facilities can occur. Parallel transmission systems, in particular those comprising of double circuit lines, are finding widespread usage [1] and better utilization of such lines in terms of increased loading is one solution to the restrictions imposed on transmission system growth. However, fault diagnosis in such systems poses many difficulties for conventional logical techniques or linear algorithms principally because a faulted phase(s) on one circuit has an affect on the sound phases (both on the remaining healthy phases of the faulted circuit and the healthy phases of the unfaulted parallel circuit) due to the mutual coupling effect

Manuscript received March 25, 1998; revised February 1, 1999 and March 29, 1999.

R. K. Aggarwal, Q. Y. Xuan, A. T. Johns, and F. Li are with the Department of Electronic and Electrical Engineering, University of Bath, Claverton Down, Bath BA2 7AY, U.K

A. Bennett is with Reyrolle Protection, Hebburn, Tyne-and-Wear NE31 1TZ, U.K.

Publisher Item Identifier S 1045-9227(99)05697-0. between the two circuits. The problem is compounded by the fact that this mutual coupling is not constant and is dependent upon a complex interplay among a number of variables such as generator source capacities, fault location, fault resistance, type of fault, etc. As a consequence, the sound phases may sometimes be wrongly diagonised as being the faulted phase(s) under certain fault conditions; this in turn can cause tripping of the wrong phase(s), thereby threatening system stability and/or continuity of power supply. There is thus a need to develop an alternative fault diagnosis technique.

Recently, artificial intelligence (AI) techniques which include neural networks (NN's), fuzzy logic, genetic algorithms, and expert systems, have been used worldwide to solve many nonlinear classification problems. Since each branch has its own individual advantages/disadvantages, for any complex classification task, it is essential to compare all possible AI techniques and then choose the one most appropriate for solving a specific problem. For example, in the case of fault classification, apart from the accuracy requirements, speed is of the essence (typically 5-10 ms) [2]. In this respect, an NN on its own or an NN integrated with fuzzy logic or genetic algorithm for training purposes can be employed; it should be mentioned that an NN on its own requires a much longer training time compared to the latter approach, but it is important to note that once trained and provided the size of the $\mathrm{NN}$ is not too large, its on-line testing and application is fast and hence a fault classifier based solely on an NN can satisfy the speed requirements. Another technique, such as that based on combined fuzzy logic and an expert system, has been found to be useful for fault detection in power systems but such an approach is not particularly well suited for fault classification [3], [4].

From the viewpoint of NN training techniques, these can be defined as supervised, unsupervised, and reinforced learning algorithms. In this respect, a typical supervised EBP network has been used as a fault classifier, which employs a nonlinear regression technique to achieve minimum global error [5]. However, this classification technique is more suited to a single-circuit transmission system rather than a more complex transmission system such as that comprising of double-circuit lines; this is so because in the case of the latter, the voltage and current waveforms (sinusoidal waveforms disturbed by a fault) are more complex under different fault conditions. Moreover, the features within the waveforms are not obvi- 
ous even through multidimensional diagrams, irrespective of how the input and output signals, the data sampling rate, and data window length have been chosen; the trained $\mathrm{NN}$ never reaches the desired global minimum and often the NN is stuck at local minimum. When the voltage and current waveforms are preprocessed into nonstationary waveforms [6], although a fault classifier with the supervised training technique can reach the desired global minimum, the classification rate is only about $79 \%$ and this is not particularly high. When a fault classifier is based on an unsupervised (i.e., Kohonen NN) or combined supervised and unsupervised training technique, such as self-organizing mapping (SOM) $\mathrm{NN}$, radial-basis function (RBF) NN, counterprogation $(\mathrm{CP})$ NN, etc., by using the nonstationary waveforms [7], [8], the classification rate can reach a high level of about $95 \%$, but the number of neurons in the Kohonen layer or prototype layer are very large. These types of NN's are thus less suitable for practical implementation of fault diagnosis in power systems simply because the calculation times involved are rather long. Reinforced learning technique has also been used in [9]; here, a genetic algorithm is used to search the weight space of a multilayer feedforward $\mathrm{NN}$ without the use of any gradient information. The basic concept behind this technique is that a complete set of weights $\left(w_{1}, w_{2}, \cdots, w_{n}\right)$ are coded into a string, which has an associated fitness finding attribute for the optimal weights. Although the reinforced learning performs a global search and therefore minimizes the possibility of getting stuck in local minima, the training is very time-consuming; classification rate is around $85 \%$, which is still not satisfactory.

For an NN to be called a fuzzy neural net (FNN), the signals and/or the weights must be fuzzy sets. In this respect, there are three different types of FNN's: the first type has real number signals but fuzzy weights; the second type has fuzzy signals and real number weights; and the third FNN has both fuzzy signals and fuzzy weights. With regard to fault diagnosis, experience has shown that the first FNN, i.e., the one based on the concept of fuzzy weights, is the one most suitable for solving the fault classification problem. In this respect, it has been found that the previously mentioned fuzzy ARTmap network rather than the more conventional FNN, enhances the performance (both in terms of convergence speed and accuracy) even further and this is the approach adopted here.

This paper proposes a fault diagnosis technique for complex transmission systems by using a fuzzy ARTmap NN. In particular, it places emphasis on describing the basic concepts of the fuzzy ARTmap and its advantages over other NN-based techniques. Also experimentation with the training of such a network is described in detail. The test results clearly show that the proposed technique is well-suited for this particular fault classification problem.

\section{FuZZY ARTMAP TechniQue}

Fuzzy ARTmap is an incremental supervised learning algorithm which combines fuzzy logic and adaptive resonance theory (ART) neural network for recognition of pattern cate-

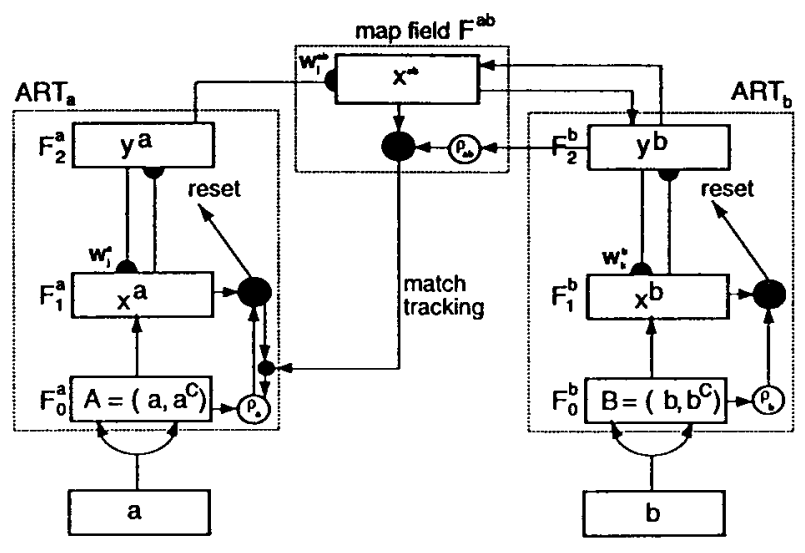

Fig. 1. A typical fuzzy ARTmap architecture.

gories and multidimensional maps in response to input vectors presented in an arbitrary order. It realizes a new minimax learning rule which conjointly minimizes predictive error and maximizes code compression, and therefore gives generalization. This is achieved by a match tracking process that increases the ART vigilance parameter (fuzzy degree of membership of the input with respect to the category templates) by the minimum amount needed to correct a predictive error (PE). The fuzzy ARTmap neural network is composed of two fuzzy ART modules, i.e., fuzzy $\mathrm{ART}_{a}$ and fuzzy $\mathrm{ART}_{b}$, which are typified in Fig. 1 and are essentially the same as those described by Carpenter et al. in [10]. The interactions mediated by the map field $F^{a b}$ may be operationally characterized as follows.

\section{A. $A R T_{a}$ and $A R T_{b}$}

Inputs to $\mathrm{ART}_{a}$ and $\mathrm{ART}_{b}$ are in the complement code form: for $\mathrm{ART} a, I=A=\left(a, a^{c}\right)$; for $\mathrm{ART}_{b}, I=B=\left(b, b^{c}\right)$ (see Fig. 1). Variables in $\mathrm{ART}_{a}$ or $\mathrm{ART}_{b}$ are designated by subscripts " $a$ " and " $b$," respectively. For $\mathrm{ART}_{a}$, let $x^{a}=\left\{x_{1}^{a} \cdots x_{2 M a}^{a}\right\}$ denote the $F_{1}^{a}$ output vector, let $\boldsymbol{y}^{a}=$ $\left\{y_{1}^{a} \cdots y_{N a}^{a}\right\}$ denote $F_{2}^{a}$, and let $\boldsymbol{w}_{j}^{a}=\left\{w_{j 1}^{a}, w_{j 2}^{a}, \cdots, w_{j 2 M a}^{a}\right\}$ denote the $j$ th $\mathrm{ART}_{a}$ weight vector. For $\mathrm{ART}_{b}$, let $\boldsymbol{x}^{b}=$ $\left\{x_{1}^{b} \cdots x_{2 M b}^{b}\right\}$ denote $F_{1}^{b}$ output vector, let $\boldsymbol{y}^{b}=\left\{y_{1}^{b} \cdots y_{N b}^{b}\right\}$ denote $F_{2}^{b}$, and let $\boldsymbol{w}_{k}^{b}=\left\{w_{k 1}^{b}, w_{k 2}^{b}, \cdots, w_{k 2 M b}^{b}\right\}$ denote the $k$ th $\mathrm{ART}_{b}$ weight vector. For the map field, let $\boldsymbol{x}^{a b}=$ $\left\{x_{1}^{a b}, \cdots, x_{N b}^{a b}\right\}$ denote the $F^{a b}$ output vector, and let $\boldsymbol{w}_{j}^{a b}=$ $\left\{w_{j 1}^{a b}, \cdots, w_{j N b}^{a b}\right\}$ denote the weight vector from the $j$ th $F_{2}^{a}$ node to $F^{a b}$. Vectors $\boldsymbol{x}^{a}, \boldsymbol{y}^{a}, \boldsymbol{x}^{b}, \boldsymbol{y}^{b}$, and $\boldsymbol{x}^{a b}$ are set to $\mathbf{0}$ between input presentations.

\section{B. Map Field Activation}

The map field $F^{a b}$ is activated whenever one of the $\mathrm{ART}_{a}$ or $\mathrm{ART}_{b}$ categories is active. If node $J$ of $F_{2}^{a}$ is chosen, then its weights $w_{j}^{a b}$ activate $F^{a b}$. If node $K$ in $F_{2}^{b}$ is active, then node $K$ in $F^{a b}$ is activated by 1-to- 1 pathways between $F_{2}^{b}$ and $F^{a b}$. If both $\mathrm{ART}_{a}$ and $\mathrm{ART}_{b}$ are active, then $F^{a b}$ becomes active only if $\mathrm{ART}_{a}$ predicts the same category as $\mathrm{ART}_{b}$ via the weights $w_{j}^{a b}$. 
The $F^{a b}$ output vector $\boldsymbol{x}^{a b}$ obeys the following:

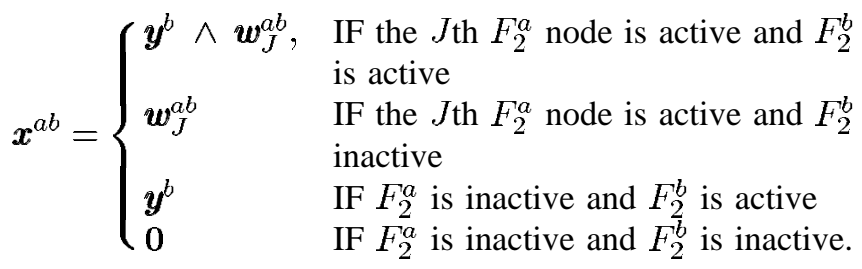

From (1), $\boldsymbol{x}^{a b}=0$ if the prediction $\boldsymbol{w}_{J}^{a b}$ is disconfirmed by $\boldsymbol{y}^{b}$. Even such a mismatch triggers an $\mathrm{ART}_{a}$ search for a better category, as follows.

\section{Match Tracking}

At the start of each input presentation, the $\mathrm{ART}_{a}$ vigilance parameter $\rho_{a}$ equals to a baseline vigilance $\rho_{a}$. The map field vigilance parameter is $\rho_{a b}$

$$
\text { IF }\left|\boldsymbol{x}^{a b}\right|<\rho_{a b}\left|\boldsymbol{y}^{b}\right|
$$

then $\rho_{a}$ is increased until it is slightly larger than $\mid A \wedge$ $\left.\boldsymbol{w}_{J}^{a}|| \boldsymbol{A}\right|^{-1}$, where $A$ is the input to $F_{1}^{a}$, in complement coding form, and

$$
\left|\boldsymbol{x}^{a}\right|=\left|A \wedge \boldsymbol{w}_{J}^{a}\right|<\rho_{a}|\boldsymbol{A}|
$$

where $J$ is the index of the active $F_{2}^{a}$ node. When this occurs, $\mathrm{ART}_{a}$ search leads either to activation of another $F_{2}^{a}$ node $J$ with

and

$$
\left|\boldsymbol{x}^{a}\right|=\left|A \wedge \boldsymbol{w}_{J}^{a}\right| \geq \rho_{a}|A|
$$

$$
\left|\boldsymbol{x}^{a}\right|=\left|\boldsymbol{y}^{b} \wedge \boldsymbol{w}_{J}^{a b}\right| \geq \rho_{a}\left|\boldsymbol{y}^{b}\right|
$$

or, if no such node exists, to the shut-down of $F_{2}^{a}$ for the remainder of the input presentation.

\section{Map Field Learning}

Learning rules determine how the map field weights $w_{j k}^{a b}$ change through time, as follows. Weights $w_{j k}^{a b}$ in $F_{2}^{a} \rightarrow F^{a b}$ paths initially satisfy

$$
w_{j k}^{a b}(0)=1 .
$$

During resonance with the $\mathrm{ART}_{a}$ category $J$ active, $\boldsymbol{w}_{J}^{a b}$ approaches the map field vector $\boldsymbol{x}^{a b}$. With fast learning, once $J$ learns to predict the $\mathrm{ART}_{b}$ category $K$, that association is permanent, i.e., $w_{J K}^{a b}=1$ for all time.

\section{TESTED SYSTEM AND PATTERNS}

\section{A. The Tested System Model}

To demonstrate the suitability of Fuzzy ARTmap network for fault diagnosis, a fuzzy ARTmap network is employed to identify the different patterns generated from the complex transmission system which is represented in Fig. 2; this is a typical 400-kV transmission system of the type encountered on the U.K. supergrid transmission network [11]. In this respect, it should be noted that in practice, a transmission system is

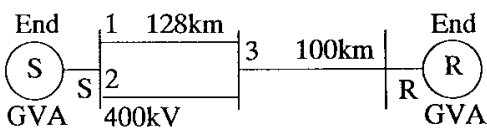

Fig. 2. The tested system model.

fully integrated whereby to each end of a line (end $S$ or $R$ ) is connected the local generation and transmission lines from other power stations within the network. However, from a viewpoint of studying the fault transient phenomena on a particular part of the network (such as of the type shown in Fig. 2), it is normal practice to lump the whole of the generation capacity, which includes local generation together with generation from other sources connected to that particular end, into one single source as though the line were terminated in one extremely large generating capacity source. Typically on the U.K. system, this can range from a maximum level of 50 gigavolt-amperes (50 GVA) to a minimum level of about 5 GVA at any particular line end. Some of the fundamental concepts of modeling a faulted transmission system have been outlined in the Appendix. A more detailed explanation can be found in [11].

Fig. 3 typifies the simulated voltage current waveform patterns at end $S$ for an " $a_{1}$ "-earth fault (i.e., " $a$ " phase fault on circuit 1) approximately $30 \mathrm{~km}$ from end $S$, where the fault classifier of the type described herein would be located in practice. All components are modeled using the wellproven and universally accepted ElectroMagnetic Transients Program (EMTP) software [12]. As can be seen, there is some high-frequency $(\mathrm{HF})$ distortion on the voltage waveforms, in particular on the faulted " $a$ " phase on fault occurrence [Fig. 3(a)]. With regard to the current waveforms, as expected, there is a large increase in the magnitude of the faulted " $a$ "phase current which is relatively distortion free; both the healthy " $b$ " and " $c$ " phase currents on circuit 1 have very small magnitudes in comparison [Fig. 3(b)]. In contrast, although the magnitudes of the current waveforms on the healthy circuit 2 are much smaller than those generated on circuit 1, the patterns impressed upon the current signals have a much higher level of HF distortion [Fig. 3(c)] and this arises principally due to the aforementioned mutual coupling effect between the two circuits.

It should be mentioned that the simulated signals attained through the EMTP software package have been extensively validated using both real data and data generated via other techniques, the frequency-domain technique for modeling the fault transient phenomena on transmission lines being one of them [11].

In practice, in a transmission network, the only signals available for fault diagnosis are the foregoing phase voltage and current waveforms. Moreover, for both measurement purposes and subsequent processing of these signals in hardware, their magnitudes have to be very significantly transduced down through suitable transducers (instrument voltage and current 

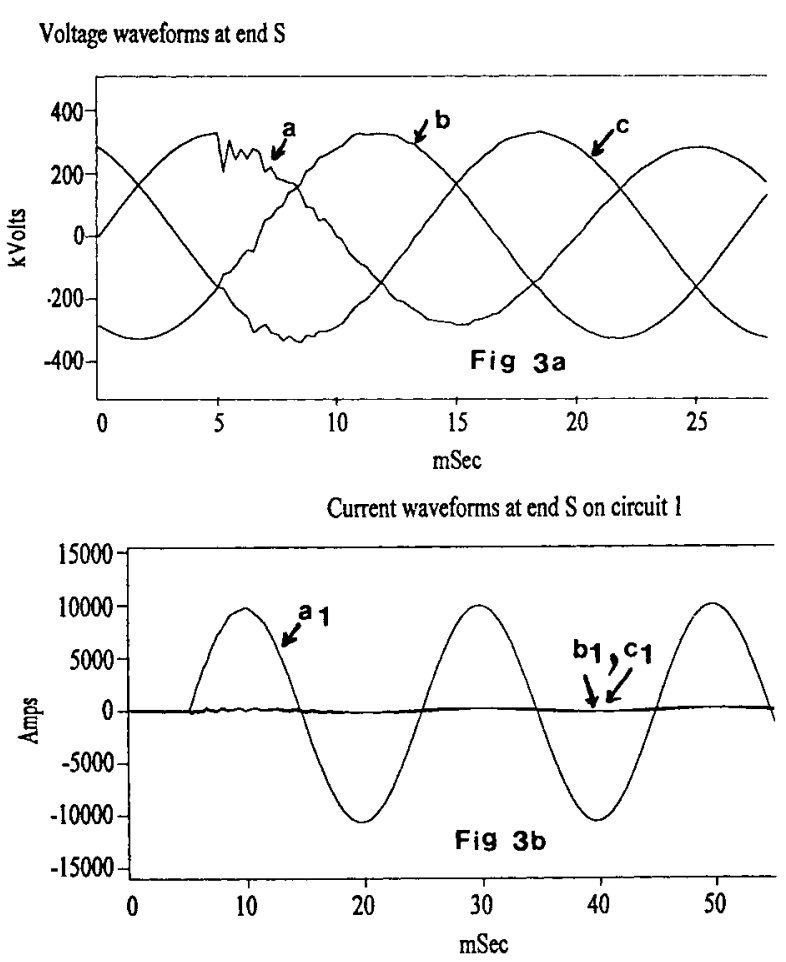

Current waveforms at end $\mathrm{S}$ on circuit 2

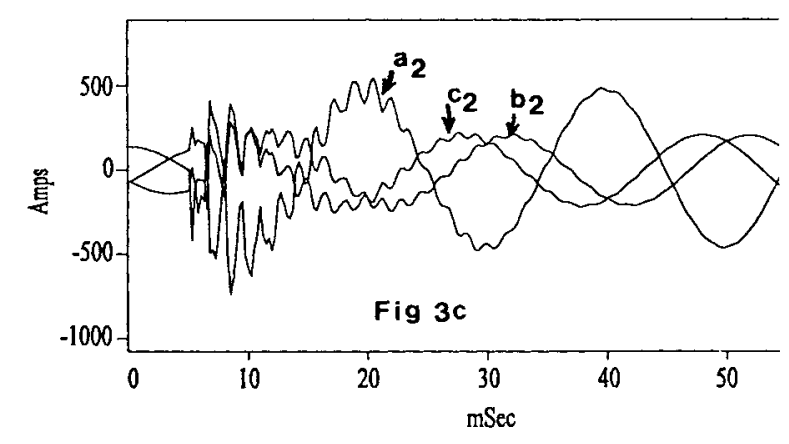

Fig. 3. Typical voltage and current patterns.

transformers more commonly known as VT's and CT's). It is thus crucial that in any computer-aided design (CAD) of the new technique, models of the VT's and CT's, analog interfaces within the digital hardware and quantization errors arising in the digitization of the analog signals should also be included in the simulation and this is the approach adopted in the fault diagnosis technique described here. Importantly, through this approach, the performance attained from the simulation studies more closely pertains to an actual practical situation than would otherwise be the case. The training patterns based on the foregoing voltages and currents are generated to cover a whole variety of commonly encountered fault types for different fault locations, fault resistances, fault inception angles, and source capacities. In this study, the training set size comprised of 250 exemplars which broadly reflects the aforementioned commonly encountered different system and fault conditions.

\section{B. Selection of Input and Output Vectors}

In order to build up an NN, the inputs and outputs of the $\mathrm{NN}$ have to be defined for pattern recognition. Here, the measured

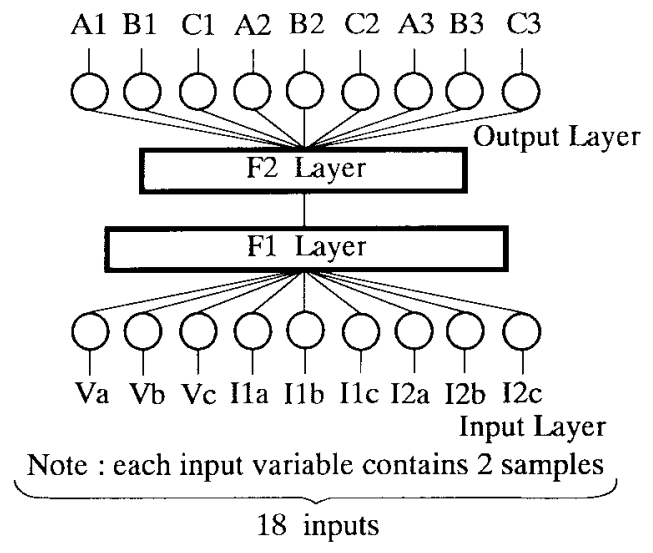

Fig. 4. The structure of the fault classifier.

three-phase voltages $\left(V_{a}, V_{b}\right.$, and $\left.V_{c}\right)$ and six-phase currents on both circuits 1 and $2\left(I_{1 a}, I_{1 b}, I_{1 c}, I_{2 a}, I_{2 b}\right.$, and $\left.I_{2 c}\right)$ which are preprocessed into nonstationary waveforms, are used as inputs to the fuzzy ARTmap network. The nine-digit outputs are composed of $A 1, B 1, C 1, A 2, B 2, C 2 A 3, B 3$, and $C 3$ in the NN, which correspond to phases $a 1, b 1, c 1$ for circuit 1 , phases $a 2, b 2, c 2$ for circuit 2 , and phases $a 3, b 3, c 3$ for circuit 3 operational states, respectively. These simply represent digits " 1 " or " 0 " which signify whether there is a fault on a phase(s) and if so, which phase(s) is involved in the fault. For example, if we get an output from the NN 1,1,0,0,0,0,0,0,0 then this constitutes an $a_{1}-b_{1}$ phase fault on circuit 1 , and so on. The NN structure of the fault classifier is shown in Fig. 4.

\section{The Fault Classifier Technique BASED ON FUZZY ARTMAP NETWORK}

The fuzzy ARTmap algorithm overcomes the drawbacks associated with the EBP algorithm. Since it is not based on the gradient descent algorithm, it does not suffer from the local minima problem, and this in turn results in rapid training. It also supports incremental learning which ensures that new events can be added to the training set at a later stage with little influence on the previous learning. This attribute is very useful for training for some contingencies, which may occur from time to time in power systems; an example is a broken conductor. In the EBP algorithm, the training has to be performed right from the beginning if there is any addition required to the training set since there is no provision for any new learning. Discussion relating to the experimentation with several parameters for the aforementioned fuzzy ARTmap network (as shown in Fig. 4) is given below; this is important in view of the fact that the values of these parameters (and therefore the optimal choice) can greatly influence the classification rate.

\section{A. The Structure of the NN}

This is composed of an input layer, layers $F 1, F 2$, and an output layer (see Fig 4); these layers are associated with either $\mathrm{ART}_{a}$ or $\mathrm{ART}_{b}$ shown in Fig. 1. The number of neurons in the input layer are 18 (two samples for each input variable), 36 in $F 1$ (double inputs), 33 in $F 2$ (these depend upon the number 
of training sets) and nine outputs. It should be mentioned that although the number of neurons in the $F 2$ layer reflect the number of category modes in each ART system shown in Fig. 1, the other training parameters (as discussed later) can also be adjusted to control the number of neurons in $F 2$ and, therefore, the size of the NN. In this study, the optimum number of $\mathrm{ART}_{a}$ categories were 30 .

\section{B. Baseline Vigilance}

For any ARTmap network, it has the vigilance parameter (also called a confidence measure) which measures whether the chosen hypothesis matches the data well enough to generate its prediction. If vigilance is low, even poor matches are acceptable. Many different examples can then be incorporated into one category so that compression and generalization are high. If vigilance is high, then even good matches may be rejected, and hypothesis testing may be initiated to select a new category. In this case, a few examples activate the same category, so that compression and generalization are low. Herein, the vigilance factor is set to 0.05 . The match-tracking system then increases the vigilance until a match is achieved.

\section{Recode Rate}

A "slow-recode" long-term memory updating scheme is used in the fuzzy ARTmap. When a category is newly formed, the long-term memory is immediately set to match the input data. Therefore, a winning category's long-term memory is only partially updated according to the recode rate which is set at 0.2 in this particular application.

\section{Choice of Parameter $(\alpha)$}

This parameter is encoded into the long-term memory. The choice function for a category PE is $X /(\alpha+|X|)$, where $X$ is the activity in the $F 1$ layer shown in Fig. 1.

\section{E. Error Tolerance}

This parameter is used by the match-tracking system for triggering a category reset which is achieved by gradually increasing the vigilance. The absolute error at the output layer is compared with the tolerance and if the tolerance is greater than the set level, a reset is triggered. Here, the error tolerance value is set to 0.01 . In essence, the error tolerance signifies a relationship between the NN "ideal" output and the actual output during training (this situation is somewhat analogous to the more traditional $\mathrm{NN}$ architecture employing the error back-propagation learning algorithm). For example, an error tolerance level set to 0.01 indicates that for a certain fault data input file, the training is complete i.e., the NN has converged, once the absolute error at the output layer has reached this threshold level.

The classification rates for fault diagnosis under different parameter selection are compared and are as shown in Table I.

It is apparent from the above table that the best classification rate is $97 \%$, which is achieved from the parameter selections of 0.005 baseline vigilance, 0.2 recode rate, 0.1 choice parameter, and 0.01 error tolerance, and these were the training
TABLE I

Classification Rate Comparison Under Different Parameter Variations

\begin{tabular}{lll}
$\begin{array}{l}\text { Choice parameter } \\
\text { rate }\end{array}$ & error tolerance & $\begin{array}{l}\text { classification } \\
\text { rate }\end{array}$ \\
\hline (a) $\alpha=2.0$ & 0.01 & $80 \%$ \\
& 0.001 & $78 \%$ \\
& 0.0001 & $65 \%$ \\
\hline (b) $\alpha=1.5$ & 0.01 & $83 \%$ \\
\hline (c) $\alpha=1.0$ & 0.01 & $94 \%$ \\
\hline (d) $\alpha=0.5$ & 0.01 & $89 \%$ \\
\hline (e) $\alpha=0.1$ & 0.01 & $97 \%$ \\
\hline (f) $\alpha=0.05$ & 0.01 & $93 \%$
\end{tabular}

parameters used in the study presented herein. However, the best classification rate by using EBP algorithm under the same training sets is only $80 \%$, no matter how the training parameters are chosen. Even in studies based on employing other NN architectures such as the Kohonen network [7] or the counterpropagation network [8], the performance achievable in terms of the fault classification rate is lower than that attained using the fuzzy ARTmap network. It is thus clearly evident that the latter, in comparison to the more traditional $\mathrm{NN}$ architectures, is much better suited for fault classification, in particular on the more complex parallel transmission systems.

This attribute of the ARTmap classifier is clearly depicted in Table II which shows its performance under a variety of different system and fault conditions; all the test results (based on data different from those employed during training of the network) indicate the correct solution. Equally importantly, the results also show stability of the technique under normal steady-state conditions and rapidly converge to the requisite values of the output variables (either very close to unity or zero) under fault conditions; this further confirms the suitability of the proposed classifier for fault type classification in complex multicircuit transmission lines. It should be mentioned that the technique described in this paper is based on a time-domain moving-window approach (each window comprises of two data samples) as discussed previously. The results in Table II show that in the fault cases presented, there is a very rapid transition in the NN outputs as the windows move from the pre-fault to the fault states (the first set of outputs in each fault case depict the prefault state, and the fault state subsequently). Significantly, the NN outputs show the correct levels (for a particular type of fault) throughout the fault period.

\section{CONCLUSIONS}

This paper proposes a novel fault diagnosis technique for complex parallel transmission systems by using fuzzy ARTmap neural network. The artificial neural network based on the supervised adaptive resonance theory can identify the faulted phase with a high degree of accuracy. Compared with the EBP algorithm, the classification rate is significantly higher and the training times required are much shorter for the same 
TABLE II

Actual Test Results Through the Fuzzy ARTmap Neural Network (Time Step $\Delta t=1.25 \mathrm{~ms}$ )

$\begin{array}{lllllllll}\mathrm{A} 1 & \mathrm{~B} 1 & \mathrm{Cl} & \mathrm{A} 2 & \mathrm{~B} 2 & \mathrm{C} 2 & \mathrm{~A} 3 & \mathrm{~B} 3 & \mathrm{C} 3\end{array}$

Case 1: normal steady state condition with source capacity at end $S$ of 35 GVA (or 50GVA)

$\left.\begin{array}{lllllllll}0.01934 & 0.00915 & 0.00000 & 0.00000 & 0.00569 & 0.00157 & 0.01924 & 0.01668 & 0.01755 \\ 0.02053 & 0.00714 & 0.00000 & 0.00000 & 0.00902 & 0.00734 & 0.02090 & 0.00000 & 0.00000 \\ 0.01844 & 0.00373 & 0.00000 & 0.00000 & 0.00647 & 0.00818 & 0.02023 & 0.01455 & 0.01347\end{array}\right\}$ normal steady state

Case 2: an a1-phase-earth fault occurring on circuit 1

$\begin{array}{lllllllll}0.01844 & 0.00373 & 0.00000 & 0.00000 & 0.00647 & 0.00818 & 0.02023 & 0.01455 & 0.01347 \rightarrow \text { normal steady state } \\ 0.97721 & 0.00774 & 0.00000 & 0.00000 & 0.00677 & 0.00358 & 0.00533 & 0.02446 & 0.02432 \rightarrow \text { fault } \\ 0.93584 & 0.00735 & 0.00000 & 0.00000 & 0.01022 & 0.00711 & 0.00443 & 0.00844 & 0.00654 \rightarrow \text { fault }\end{array}$

case 3: a b2-phase-earth fault occurring on circuit 2

$\begin{array}{lllllllll}0.01844 & 0.00373 & 0.00000 & 0.00000 & 0.00647 & 0.00818 & 0.02023 & 0.01455 & 0.01347 \rightarrow \text { normal steady state } \\ 0.01826 & 0.00439 & 0.00000 & 0.00000 & 0.96728 & 0.00910 & 0.00874 & 0.00773 & 0.00774 \rightarrow \text { fault } \\ 0.02059 & 0.00183 & 0.00221 & 0.00151 & 0.96501 & 0.00742 & 0.00886 & 0.01663 & 0.02341 \rightarrow \text { fault }\end{array}$

Case 4: a1-phase to b1- phase fault occurring on circuit 1

$\begin{array}{lllllllll}0.01844 & 0.00373 & 0.00000 & 0.00000 & 0.00647 & 0.00818 & 0.02023 & 0.01455 & 0.01347 \rightarrow \text { normal steady state } \\ 0.90442 & 0.92554 & 0.00122 & 0.00225 & 0.02244 & 0.02478 & 0.00667 & 0.00445 & 0.00881 \rightarrow \text { fault } \\ 0.98642 & 0.97448 & 0.00000 & 0.00000 & 0.01233 & 0.01785 & 0.00556 & 0.00887 & 0.00774 \rightarrow \text { fault }\end{array}$

Case 5: a1-phase to b2-phase intercircuit fault occurring on circuits 1 ans 2

$\begin{array}{lllllllll}0.01844 & 0.00373 & 0.00000 & 0.00000 & 0.00647 & 0.00818 & 0.02023 & 0.01455 & 0.01347 \rightarrow \text { normal steady state }\end{array}$

$\begin{array}{llllllllll}0.99771 & 0.00877 & 0.00133 & 0.00442 & 0.93341 & 0.00000 & 0.00000 & 0.00776 & 0.00332 \rightarrow \text { fault }\end{array}$

$\begin{array}{llllllllll}0.97741 & 0.00000 & 0.00000 & 0.00000 & 0.95532 & 0.00122 & 0.22300 & 0.02456 & 0.00177 \rightarrow \text { fault }\end{array}$

Case 6: a1, b1, c1 three phase-earth fault occurring on circuit 1

$\begin{array}{lllllllll}0.01844 & 0.00373 & 0.00000 & 0.00000 & 0.00647 & 0.00818 & 0.02023 & 0.01455 & 0.01347 \rightarrow \text { normal steady state } \\ 0.98773 & 0.90881 & 0.92110 & 0.00245 & 0.00345 & 0.00122 & 0.00445 & 0.00553 & 0.00000 \rightarrow \text { fault } \\ 0.97881 & 0.93522 & 0.97205 & 0.00000 & 0.00000 & 0.02376 & 0.01568 & 0.00000 & 0.00000 \rightarrow \text { fault }\end{array}$

Case 7: b3, c3 phase-earth fault occurring on circuit 3

$\begin{array}{lllllllll}0.01844 & 0.00373 & 0.00000 & 0.00000 & 0.00647 & 0.00818 & 0.02023 & 0.01455 & 0.01347 \rightarrow \text { normal steady state } \\ 0.00000 & 0.00000 & 0.01144 & 0.00884 & 0.02389 & 0.00889 & 0.00000 & 0.96873 & 0.94458 \rightarrow \text { fault } \\ 0.00224 & 0.00331 & 0.00000 & 0.00000 & 0.00943 & 0.00448 & 0.00445 & 0.90443 & 0.96443 \rightarrow \text { fault }\end{array}$

training sets. Furthermore, the fuzzy ARTmap network can be improved by adding more events to it, such as contingencies, without having to completely retrain the network. In particular, some experimentation in the training of such a network is given in the paper in detail. All the test results presented show that the proposed fault diagnosis technique based on fuzzy ARTmap network is well suited for the complex transmission systems than other more conventional NN-based techniques.

\section{APPENDIX}

\section{A. A Faulted Transmission System}

A high-voltage transmission line is prone to faults due to natural hazards. In this respect, the most common fault 


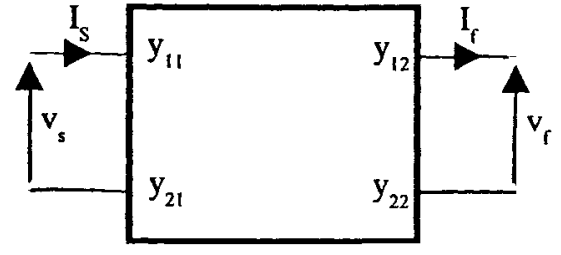

Fig. 5. Two-port transfer matrix of the line.

(over 90\%) is of the single-phase-ground type principally caused by lightning strikes; the latter raises the potential of a phase conductor (normally insulated from the tower which is connected to ground) to such a high level as to cause a flashover of the conductor to the tower and this results in a ground fault. Other, less common, types of fault involve two phases such as: phase-phase caused by conductors clashing due to high winds and phase-phase-ground caused by an object (such as a tree) falling on to a line. A fault constitutes a sudden circuit change and sets up the travelingwave phenomenon on the transmission line, which manifests itself as high-frequency distortion on the normal steady-sate power frequency voltage and current waveforms, as depicted in Fig. 3.

\section{B. Fundamental Relationships}

Any multiconductor transmission line section is defined by its series impatience matrix per unit length $Z$ and the corresponding shunt admittance matrix $Y$. Each element of $Z$ varies with frequency and is determined by the conductor types, their physical geometry and the nature of the earth plane.

The theory of natural modes developed by Wedepohl [11] enables a solution to the system voltage steady-state equations given by (7) to be transformed into a series of independent differential equations of the form given in (8)

$$
\begin{aligned}
d^{2} V / d x^{2} & =Z Y V \\
V & =\exp (-\psi x) V_{i}+\exp (\psi x) V_{r}
\end{aligned}
$$

where $\psi=Q \gamma Q^{-1}, Q$ is the voltage eigenvector matrix, and $\gamma$ is the propagation constant matrix.

Matrix function theory permits easy evaluation of the hyperbolic functions, the polyphase surge impedance, and admittance necessary for a solution to the problem. For example, the polyphase surge admittance matrix is given by

$$
Y_{o}=\left(Q \gamma^{-1} Q^{-1} Z\right)^{-1} \text {. }
$$

1) Transmission Line Transfer-Matrix Function: A faulted transmission system essentially consists of a network of cascaded sections. Two-port transfer matrices are particularly useful in the solution of such a problem. For example, with reference to Fig. 5, the transfer matrix representing a line section, say up to the fault point, is given by

$$
\left[\begin{array}{l}
I_{s} \\
V_{r}
\end{array}\right]=\left[\begin{array}{ll}
y_{11} & y_{12} \\
y_{21} & y_{22}
\end{array}\right]\left[\begin{array}{l}
V_{a} \\
V_{r}
\end{array}\right]
$$

where $y_{11}=Y_{0} \operatorname{coth}(\psi x), y_{12}=Y_{0} \operatorname{cosech}(\psi x)$.

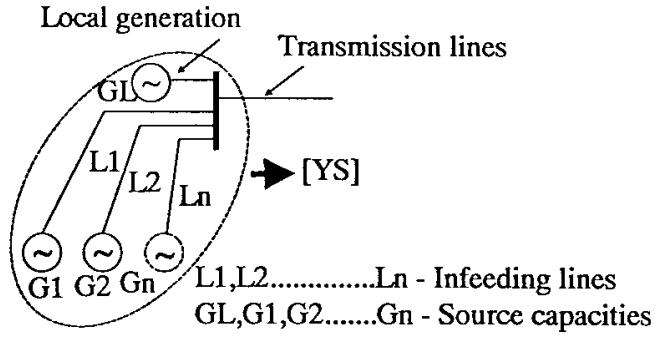

Fig. 6. General source network.

2) Source-Side Network Matrix: The source network considered here at each terminating busbar is a general source model comprising of some local generation and a number of infeeding parallel lines from other power stations, each with its own generation, all the generations being based upon arbitrarily defined short-circuit levels. This is shown in Fig 6 and when lumped together, forms the total source capacity (in GVA at any busbar) as referred to in Fig. 2.

It is relatively easy to define an equivalent source admittance matrix [YS] at each terminating busbar and this is then used in combination with the corresponding transmission line admittance matrices of (10) to form the full transmission line fault transient model.

\section{REFERENCES}

[1] M. I. Gilany, O. P. Malik, and G. S. Hope, "A laboratory investigation of a digital protection technique for parallel transmission lines," IEEE Trans. Power Delivery, vol. 10, pp. 187-193, Jan. 1995.

[2] A. D. U. Thompson and C. Bijoy, "An accurate fault classification technique for power system monitoring devices," in IEEE-Proc. Fault Monitoring, 1994, pp. 1344-1349.

[3] A. Ferrero, S. Sangiovanni, and E. Zappitelli, "A fuzzy-set approach to fault-type identification digital relaying," IEEE Trans. Power Delivery, vol. 10, pp. 169-175, Jan. 1995.

[4] M. Enns, L. Budler, T. W. Cease, A. Elneweihi, E. Guro, M. Kezunovic, J. Linders, P. Leblanc, J. Postforoosh, R. Ramaswami, F. Soudi, R. Taylor, H. Ungrad, S. S. Venkata, and J. Zipp, "Potential applications of expert systems to power system protection," IEEE Trans. Power Delivery, vol. 9, pp. 720-728, Apr. 1994

[5] A. T. Johns, Q. Y. Xuan, and Y. H. Song, "Adaptive distance protection technique for controllable series compensated EHV transmission systems," in Proc. IPEC'93, Singapore, 1993.

[6] Q. Y. Xuan, R. K. Aggarwal, and A. T. Johns, "AI based adaptive protection scheme for double-circuit lines," in Proc. Int. ICSC Symp. Soft Computing (SOCO'96), Univ. Reading, U.K., Mar. 1996, pp. 126-130.

[7] R. K. Aggarwal, Q. Y. Xuan, and A. T. Johns, "Fault classification for double-circuit lines using self-organization mapping neural network," in Proc. 32nd UPEC, Sept. 1997, pp. 440-443.

[8] Q. Y. Xuan, R. K. Aggarwal, and A. T. Johns, "Counterpropagation network based fault classification for double-circuit lines," in Melecon'96 8th Mediterranean Electrotechnical Conf., Bari, Italy, May 1996, pp. $657-660$.

[9] Y. H. Song, A. T. Johns, Q. Y. Xuan, and J. Y. Liu, "Genetic algorithm based NN applied to fault detection for EHV transmission systems" in Proc. 6th IEE Int. Conf DPSP, Apr. 1997, no. 434, pp. 278-281.

[10] G. A. Carpenter, S. Grossberg, N. Markuzon, J. H. Reynolds, and D. B Rosen, "Fuzzy ARTMAP: A neural network architecture for incremental supervised learning of analog multidimensional maps," IEEE Trans. Neural Networks, vol. 3, pp. 698-705, Sept. 1992.

[11] A. T. Johns and R. K. Aggarwal, "Digital simulation of faulted ehv transmission lines with particular reference to very high speed protection," Proc. Inst. Elect. Eng., vol. 123, no. 4, Mar. 1976.

[12] W. S. Meyer and H. W. Dommel, "Numerical modeling of frequency dependent transmission line parameters in an electro-magnetic transients programme," IEEE Trans. Power Apparat. Syst., vol. PAS-93, 1974. 
Raj K. Aggarwal (SM'91) received the B.Eng. and Ph.D. degrees from the University of Liverpool, U.K., in 1970 and 1973, respectively.

He joined the Power and Energy Systems Group at the University of Bath, U.K., where he is now a Reader. His main areas of interest are power system modeling and the application of digital technology and artificial intelligence to protection and control. He has published over 200 technical papers,

Dr. Aggarwal is a fellow of the IEE (U.K.).

Q. Y. Xuan received the B.Sc. degree in electrical and electronic engineering from Chengdu University of Science and Technology, China, in 1987, and the Ph.D. degree form Liverpool John Moors University and the University of Bath, U.K. in June 1995.

She worked in industry for five years. Presently, she is a Research Officer at the University of Bath. Her interests are in the application of artificial intelligence to power system optimization, protection, and control.

Allan T. Johns (M'88-SM' 88) received the B.Sc. and Ph.D. degrees from the University of Bath, U.K., and in 1982 was awarded the D.Sc. degree for an original and substantial contribution to knowledge in electrical engineering.

$\mathrm{He}$ is currently Professor of Electrical Engineering in the Department of Electronic and Electrical Engineering, University of Bath. He is the author of over 250 papers in the area of Electrical Power Systems.

Dr. Johns is a fellow of the IEE (U.K.).
Furong Li was born in China. She received the B.Eng. degree in electrical engineering from Hohai University, China, in 1990, and the Ph.D. degree in 1997 for work on "Application of Genetic Algorithms to Optimal Operation of Electric Power Systems."

She took up a lectureship in the Electrical Power and Energy Systems Group at the University of Bath, U.K. Her major research interest is in the area of artificial intelligence techniques to clean, economic, and secure power system operation.

Allen Bennett is the Research Manager of Reyrolle Protection and has attained considerable experience of protection design during his 30 years association with the Company. He is the U.K. representative on CIGRE 34.02 Adaptive Ptotection working group. 